\title{
Search for neutral Higgs bosons decaying into four taus at LEP2
}

\section{The ALEPH collaboration}

\section{E-mail: Roberto.Tenchini@cern.ch}

ABSTRACT: A search for the production and non-standard decay of a Higgs boson, h, into four taus through intermediate pseudoscalars, a, is conducted on $683 \mathrm{pb}^{-1}$ of data collected by the ALEPH experiment at centre-of-mass energies from 183 to $209 \mathrm{GeV}$. No excess of events above background is observed, and exclusion limits are placed on the combined production cross section times branching ratio, $\xi^{2}=\frac{\sigma\left(\mathrm{e}^{+} \mathrm{e}^{-} \rightarrow \mathrm{Zh}\right)}{\sigma_{\mathrm{SM}}\left(\mathrm{e}^{\mathrm{e}^{-}} \mathrm{e}^{-} \rightarrow \mathrm{Zh}\right)} \times B(\mathrm{~h} \rightarrow \mathrm{aa}) \times B(\mathrm{a} \rightarrow$ $\left.\tau^{+} \tau^{-}\right)^{2}$. For $m_{\mathrm{h}}<107 \mathrm{GeV} / c^{2}$ and $4<m_{\mathrm{a}}<10 \mathrm{GeV} / c^{2}, \xi^{2}>1$ is excluded at the $95 \%$ confidence level.

KeYwords: e+-e- Experiments

ARXIV EPRINT: 1003.0705 


\section{Contents}

1 Introduction 1

2 The ALEPH detector 2

3 Signal and background generation 3

4 Event selection 3

$4.1 \mathrm{Z} \rightarrow \ell^{+} \ell^{-} \quad 5$

$4.2 \mathrm{Z} \rightarrow \nu \bar{\nu}$

$\begin{array}{lll}4.3 & \text { Signal efficiency } & 7\end{array}$

$\begin{array}{llr}5 & \text { Systematic uncertainties } & 8\end{array}$

$\begin{array}{llr}6 & \text { Results } & 10\end{array}$

$\begin{array}{lll}7 & \text { Conclusions } & 11\end{array}$

\section{Introduction}

Searches conducted at LEP2 have excluded the standard model (SM) Higgs boson decaying into $\mathrm{b} \overline{\mathrm{b}}$ or $\tau^{+} \tau^{-}$for masses below $114.4 \mathrm{GeV} / c^{2}$ [1]. The LEP experiments observed a small excess in the $\mathrm{b} \overline{\mathrm{b}}$ final state for a Higgs boson mass around $100 \mathrm{GeV} / c^{2}$, which is consistent with background fluctuations or SM-like production with a reduced branching ratio into $\mathrm{b} \overline{\mathrm{b}}[2,3]$. This excess, the mild tension with electroweak precision tests [4], and the fine-tuning needed in the minimal supersymmetric standard model (MSSM) have prompted the consideration of models with exotic Higgs boson decays, such as those of the next-to-minimal supersymmetric standard model (NMSSM) $[5,6]$ as well as more general frameworks $[7,8]$. In these models, new decay channels can dominate over $h \rightarrow b \bar{b}$ and render the Higgs boson "invisible" for conventional searches. In particular, a Higgs boson decaying into two light pseudoscalars is well motivated by these models and results in a four-body final state as the pseudoscalars decay into light fermions. Scenarios with a dominant $\mathrm{h} \rightarrow 2 \mathrm{a} \rightarrow 4 \mathrm{~b}$ decay are constrained by the LEP experiments for mass up to $110 \mathrm{GeV} / c^{2}[2,9,10]$. A general search for $\mathrm{h} \rightarrow$ aa with $\mathrm{a} \rightarrow \mathrm{gg}, \mathrm{c} \overline{\mathrm{c}}, \tau^{+} \tau^{-}$was performed by the OPAL Collaboration [11], but the analysis was restricted to a Higgs boson mass, $m_{\mathrm{h}}$, in the range $45-86 \mathrm{GeV} / c^{2}$. A search for $\mathrm{h} \rightarrow 2 \mathrm{a} \rightarrow 2 \mu 2 \tau$ was recently reported by the D0 Collaboration, which resulted in limits that are a factor of 1-4 larger than the expected production cross section assuming branching ratios of the pseudoscalar as predicted by the NMSSM [12]. In this paper, a search using ALEPH data is presented for $\mathrm{h} \rightarrow 2 \mathrm{a} \rightarrow 4 \tau$ up to $m_{\mathrm{h}} \approx 110 \mathrm{GeV} / c^{2}$. 


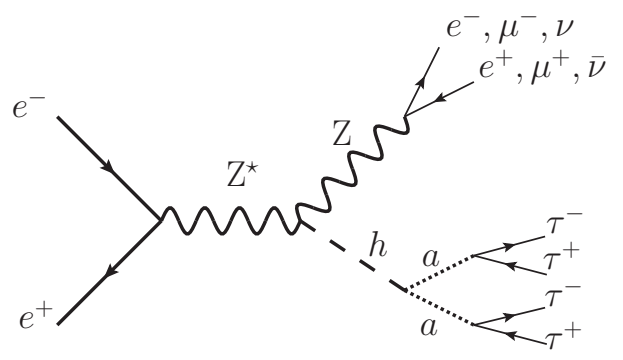

Figure 1. Higgs boson production and decay modes considered in this analysis.

The pseudoscalar a may arise from a two Higgs doublet model, as in the MSSM, or it can include a component from an additional singlet field as in the NMSSM. These possibilities differ in their details and relations between model parameters. The present search is performed in a model-independent manner and simply adopts the two main characteristics of the pseudoscalar: the coupling to a Higgs boson resulting in $h \rightarrow$ aa decay and the coupling to SM fermions proportional to their Yukawa couplings. The present analysis concentrates on the region $2 m_{\tau}<m_{\mathrm{a}} \lesssim 2 m_{\mathrm{b}}$, where the a $\rightarrow \tau^{+} \tau^{-}$decay mode is expected to be substantial. The Higgs boson production mode considered here is the Higgsstrahlung process, shown in figure 1 with $\mathrm{Z} \rightarrow \mathrm{e}^{+} \mathrm{e}^{-}, \mu^{+} \mu^{-}, \nu \bar{\nu}$.

This paper is organized as follows. The ALEPH detector is described in section 2 . Simulation of background and signal processes are described in section 3 . The event selection criteria are described in section 4 . Systematic uncertainties are discussed in section 5 . Finally, results and conclusions are presented in sections 6 and 7, respectively.

\section{The ALEPH detector}

A detailed description of the ALEPH detector can be found in ref. [13] and of its performance in ref. [14]. Charged particles are detected in the central part, which consists of a precision silicon vertex detector, a cylindrical drift chamber and a large time projection chamber (TPC), together measuring up to 31 space points along the charged particle trajectories. A $1.5 \mathrm{~T}$ axial magnetic field is provided by a superconducting solenoidal coil. Charged particle transverse momenta are reconstructed with a $1 / p_{T}$ resolution of $\left(6 \times 10^{-4} \bigoplus 5 \times 10^{-3} / p_{T}\right)(\mathrm{GeV} / c)^{-1}$. The charged particle tracks used in the present analysis (and simply called tracks) are reconstructed with at least four hits in the TPC, and originate from within a cylinder of length $20 \mathrm{~cm}$ and radius $2 \mathrm{~cm}$ coaxial with the beam and centred at the nominal collision point.

Electrons and photons are identified by the characteristic longitudinal and transverse development of the associated showers in the electromagnetic calorimeter, a 22-radiationlength thick sandwich of lead planes and proportional wire chambers with fine readout segmentation. A relative energy resolution of $0.18 / \sqrt{E}(E$ in $\mathrm{GeV})$ is achieved for isolated electrons and photons.

Muons are identified by their characteristic penetration pattern in the hadron calorimeter, a $1.2 \mathrm{~m}$ thick yoke interleaved with 23 layers of streamer tubes, together with two 


\begin{tabular}{|c|c|c|c|c|c|c|c|c|}
\hline$E_{\mathrm{CM}}(\mathrm{GeV})$ & 182.65 & 188.63 & 191.58 & 195.52 & 199.52 & 201.62 & 204.86 & 206.53 \\
\hline $\int \mathcal{L} d t\left(\mathrm{pb}^{-1}\right)$ & 56.8 & 174.2 & 28.9 & 79.9 & 86.3 & 41.9 & 81.4 & 133.2 \\
\hline
\end{tabular}

Table 1. Integrated luminosities collected at the different average centre-of-mass energies.

surrounding double-layers of muon chambers. In association with the electromagnetic calorimeter, the hadron calorimeter also provides a measurement of the hadronic energy with a relative resolution of $0.85 / \sqrt{E}(E$ in $\mathrm{GeV})$.

The total visible energy is measured with an energy-flow reconstruction algorithm which combines all the above measurements [14]. The relative resolution on the total visible energy is $0.60 / \sqrt{E}$ for high multiplicity final states. In addition to the total visible-energy measurement, the energy-flow reconstruction algorithm also provides a list of reconstructed objects, classified as charged particles, photons and neutral hadrons, and called energyflow objects in the following. These energy-flow objects are the basic entities used in the present analysis.

Below polar angles of $12^{\circ}$ and down to $34 \mathrm{mrad}$ from the beam axis, the acceptance is closed at both ends of the experiment by the luminosity calorimeter (LCAL) and a tungstensilicon calorimeter (SICAL) originally designed for the LEP 1 luminosity measurement.

The average centre-of-mass energies at which the machine operated and the corresponding integrated luminosities used in this analysis are presented in table 1.

\section{Signal and background generation}

Both signal and background were generated for all centre-of-mass energies shown in table 1 using the GEANT3-based simulation of ALEPH [15]. Backgrounds were generated with a variety of generators listed in table 2. The $\gamma \gamma$ initiated and Bhabha samples are 10-30 times larger than the data sample while others are 300-1000 times larger. The contribution to the We $\nu$ process from electrons emitted close to the beam axis is not included in KORALW and is generated with PYTHIA. The HZHA03 generator [16] was used to generate 3000 signal events (with $\mathrm{h} \rightarrow$ aa followed by a $\rightarrow \tau^{+} \tau^{-}$) for each of the three $\mathrm{Z}$ decay channels considered and for each combination of Higgs boson and pseudoscalar masses in the ranges $70<m_{\mathrm{h}}<114 \mathrm{GeV} / c^{2}$ and $4<m_{\mathrm{a}}<12 \mathrm{GeV} / c^{2}$ in steps of $2 \mathrm{GeV} / c^{2}$.

\section{Event selection}

A detailed description of the event selection criteria for the different $\mathrm{Z}$ decays, namely $\mathrm{Z} \rightarrow \mathrm{e}^{+} \mathrm{e}^{-}, \mathrm{Z} \rightarrow \mu^{+} \mu^{-}$, and $\mathrm{Z} \rightarrow \nu \bar{\nu}$ is presented below. The event topology is discussed first, followed by an explanation of how the visible decay products of the taus were treated. The $\mathrm{Z}$ reconstruction algorithm is then described and, finally, a detailed list of the cuts employed in the analysis is given.

For the mass range considered, the Higgs boson is produced approximately at rest, and thus the decay $\mathrm{h} \rightarrow 2 \mathrm{a} \rightarrow 4 \tau$ results in a pair of taus recoiling against another pair of taus. The JADE algorithm $[26,27]$ was employed to cluster into jets all energy-flow objects except 


\begin{tabular}{|c|c|c|}
\hline Category & Process & Software \\
\hline \multirow{4}{*}{$2 \mathrm{f}$} & $\mathrm{e}^{+} \mathrm{e}^{-} \rightarrow \mathrm{Z} / \gamma^{*} \rightarrow \mathrm{q} \overline{\mathrm{q}}(\gamma)$ & KK 4.14 [21] \\
& Bhabha and $\mathrm{e}^{+} \mathrm{e}^{-} \rightarrow \mathrm{Z} / \gamma^{*} \rightarrow \mathrm{e}^{+} \mathrm{e}^{-}(\gamma)$ & BHWIDE $1.01[22]$ \\
& $\mathrm{e}^{+} \mathrm{e}^{-} \rightarrow \mathrm{Z} / \gamma^{*} \rightarrow \mu^{+} \mu^{-}(\gamma)$ & KK 4.14 [21] \\
& $\mathrm{e}^{+} \mathrm{e}^{-} \rightarrow \mathrm{Z} / \gamma^{*} \rightarrow \tau^{+} \tau^{-}(\gamma)$ & KK 4.14 [21] \\
& $\mathrm{e}^{+} \mathrm{e}^{-} \rightarrow \mathrm{Z} \rightarrow \nu \bar{\nu}(\gamma)$ & PYTHIA $6.1[17]$ \\
\hline \multirow{3}{*}{$4 \mathrm{f}$} & $\mathrm{e}^{+} \mathrm{e}^{-} \rightarrow \mathrm{Z} / \gamma^{*} \rightarrow \mathrm{W}^{+} \mathrm{W}^{-}$ & KORALW 1.51 [23] \\
& $\mathrm{e}^{+} \mathrm{e}^{-} \rightarrow \mathrm{ZZ}$ & PYTHIA 6.1 [17] \\
& $\mathrm{e}^{+} \mathrm{e}^{-} \rightarrow \mathrm{Z} \mathrm{e}^{+} \mathrm{e}^{-}$ & PYTHIA 6.1 [17] \\
& $\mathrm{e}^{+} \mathrm{e}^{-} \rightarrow \mathrm{Z} \nu \bar{\nu}$ & PYTHIA 6.1 [17] \\
\hline$\gamma \gamma$ & $\mathrm{e}^{+} \mathrm{e}^{-} \rightarrow \mathrm{W}^{ \pm} \mathrm{e}^{\mp} \nu$ & PYTHIA 6.1 [17] \\
\hline $\mathrm{n} \gamma$ & $\gamma \gamma \rightarrow \ell^{+} \ell^{-}$ & PHOT02 [24, 25] \\
& $\gamma \gamma \rightarrow \mathrm{q} \overline{\mathrm{q}}$ & PHOT02 [24, 25] \\
\hline
\end{tabular}

Table 2. Details on SM background processes and their categorisation. Fragmentation, hadronisation and final state radiation were simulated with PYтніA 6.1 [17]. Рнотоs [18] was used to model final state radiation, and TAUOLA [19] was used for tau decays. More details can be found in ref. [20].

for those identified as energetic, isolated photons, energy deposits in the LCAL and SICAL, and the two hardest, oppositely-charged leptons in the case of the $\mathrm{Z} \rightarrow \ell^{+} \ell^{-}$channels. Given that each jet is expected to arise from the on-shell decay a $\rightarrow \tau^{+} \tau^{-}$, an effective way to target the signal topology is to use the JADE algorithm with $y_{\text {cut }}$ chosen to merge protojets up to a mass of $m_{\text {jet }}=15 \mathrm{GeV} / c^{2}$. This technique allows for an accurate clustering of the two tau pairs separately, thus rendering individual tau identification unnecessary and making track-multiplicity an effective discriminator against background.

Because the taus from the same a decay are highly collimated, the identification of jets containing the decay products of two taus was based only on the track multiplicity of the jets, denoted $n_{i}^{\text {track }}$, with the index $i$ ordered in decreasing jet energy. Because the tau predominantly decays either to one charged particle ("one-prong" decay) or three charged particles ("three-prong" decay), each jet is expected to contain two, four, or six tracks. To maximize the tracking efficiency, the jets were required to be well contained in the tracking volume. No distinction was made between leptonic and hadronic decays.

The $\mathrm{Z} \rightarrow \ell^{+} \ell^{-}$decay is often accompanied by additional photons from final state radiation, which can carry substantial momentum. An object is considered as an isolated photon if it is identified as a photon by the energy-flow algorithm, has $E>10 \mathrm{GeV}$, and contains less than $5 \%$ of the visible energy of the event in a cone of $10^{\circ}$ around it. The photon was considered part of the candidate $\mathrm{Z}$ system when the invariant mass of the $\ell^{+} \ell^{-} \gamma$ system was closer to the $\mathrm{Z}$ mass than the invariant mass of the lepton pair alone. This algorithm resulted in an increase of $\sim 20 \%$ in the signal efficiency after the $\mathrm{Z}$ mass window cut, $80<m_{\mathrm{Z}}<102 \mathrm{GeV} / c^{2}$.

For each of the channels below, a loose selection and final selection are presented. 
The loose selection isolates the broad characteristics of the signal events and allows for comparison of the data and simulated backgrounds.

\section{1 $\mathrm{Z} \rightarrow \ell^{+} \ell^{-}$}

The loose selection consisted of the following requirements. An $\mathrm{e}^{+} \mathrm{e}^{-}$or $\mu^{+} \mu^{-}$pair and the presence of two jets (or 3 jets with $n_{3}^{\text {track }} \leq 2$ ) were required for consistency with the final state of the signal. The three-jet events are kept to recover signal efficiency for events with converted photon arising from final state radiation. Proper containment of the jet in the tracking volume was ensured by requiring $\left|\cos \theta_{\mathrm{j}_{1}}\right|<0.9$ and $\left|\cos \theta_{\mathrm{j}_{2}}\right|<0.9$, where $\theta_{\mathrm{j}_{i}}$ is the angle of the $i^{\text {th }}$ jet with respect to the beam axis . Additional lepton isolation was imposed by requiring that a cone of $10^{\circ}$ around each lepton contained less than $5 \%$ of the visible energy of the event and $\cos \theta_{\mathrm{j} 1}^{\min }<0.95$, where $\theta_{\mathrm{j} 1}^{\min }$ is the minimum angle between each pairing of a jet and lepton.

The final selection consisted of the following requirements and maintained an acceptable signal efficiency while rejecting most backgrounds. A mass window for the candidate $\mathrm{Z}$ between $80-102 \mathrm{GeV} / c^{2}$ was effective at removing two-fermion backgrounds. Due to the neutrinos from tau decays the signal was separated from fully hadronic final states by requiring $\mathbb{E}>20 \mathrm{GeV}$, where $\mathbb{E}$ is the missing energy in the event. The expected jet configuration of the signal was enforced by requiring $\cos \theta_{\mathrm{j}_{1} \mathrm{j}_{2}}<0$, where $\theta_{\mathrm{j}_{1} \mathrm{j}_{2}}$ is the angle between the two jets. Finally, the remaining backgrounds were suppressed by requiring $n_{1,2}^{\text {track }}=2$ or 4 , the dominant track multiplicities expected in the signal. Figures 2 and 3 show the distribution of the reconstructed $\mathrm{Z}$ mass and missing energy for the $\mathrm{Z} \rightarrow \mathrm{e}^{+} \mathrm{e}^{-}$and $\mathrm{Z} \rightarrow \mu^{+} \mu^{-}$channels, respectively. The numbers of events passing loose and final selection in data and simulated background are shown in table 3 .

\section{$4.2 \quad \mathrm{Z} \rightarrow \nu \bar{\nu}$}

All objects found in the event were clustered into jets as described above. The loose selection consisted of the following requirements. Missing energy greater than $30 \mathrm{GeV}$ and missing mass, $\not h$, greater than $20 \mathrm{GeV} / c^{2}$ were used to reject dijet and other twofermion backgrounds. In order to further reject the $\gamma \gamma$ background, events were required to have $E_{\mathrm{vis}}>0.05 E_{\mathrm{CM}}$ and $\left|\cos \theta_{\mathrm{me}}\right|<0.9$, where $E_{\mathrm{vis}}$ is the visible energy and $\theta_{\mathrm{me}}$ is the angle between the missing momentum vector and the beam axis. Events were required to have two well-contained jets with $\left|\cos \theta_{\mathrm{j}}\right|<0.85$, dijet invariant mass $m_{\mathrm{j}_{1} \mathrm{j}_{2}}>10 \mathrm{GeV} / c^{2}$, dijet angular separation $\cos \theta_{\mathrm{j}_{1} \mathrm{j}_{2}}<0$, and the highest energy jet was required to have $E_{\mathrm{j}_{1}}>25 \mathrm{GeV}$ and $n_{1}^{\text {track }}=2$ or 4 .

The final selection consisted of the following requirements. First, the requirement $E_{\mathrm{j}_{1}}+E_{\mathrm{j}_{2}}+\mathbb{E}>E_{\mathrm{CM}}-5 \mathrm{GeV}$ was used to reject events with energy deposits in the forward regions of the detector. Consistency with $\mathrm{Z} \rightarrow \nu \bar{\nu}$ was ensured by requiring $\mathbb{E}>60 \mathrm{GeV}$ and $\not h>90 \mathrm{GeV} / c^{2}$. The distribution of aplanarity for the signal is strongly peaked near 0 , while the remaining backgrounds have a longer tail. The tail of the aplanarity distribution for the signal extends further for larger $m_{\mathrm{a}}$ and smaller $m_{\mathrm{h}}$; larger $m_{\mathrm{a}}$ leads to broader jets and lighter Higgs bosons can be produced with more momentum reducing the opening angle between the jets in the laboratory frame. Thus the requirement aplanarity $<0.05$ 


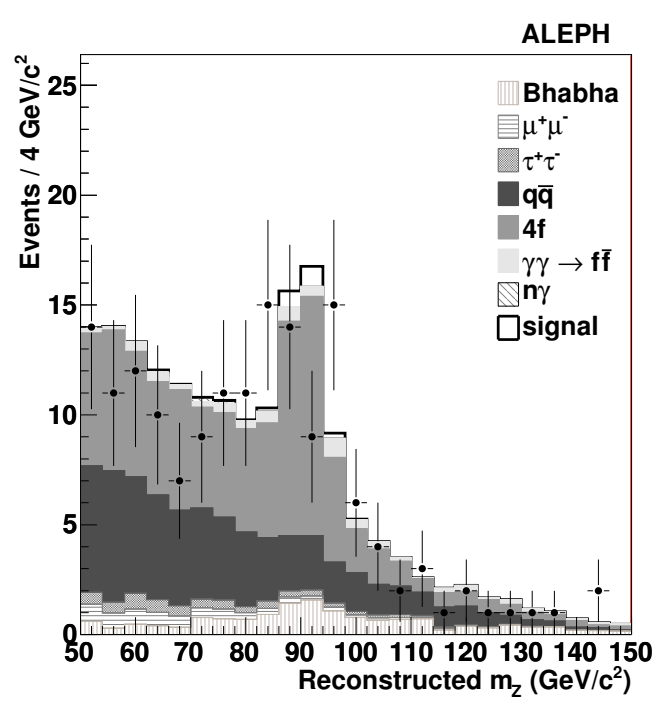

(a)

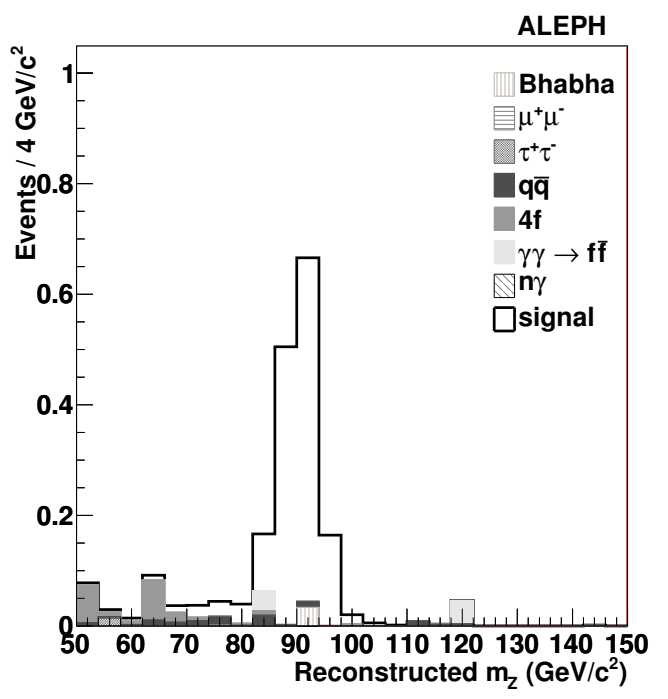

(c)

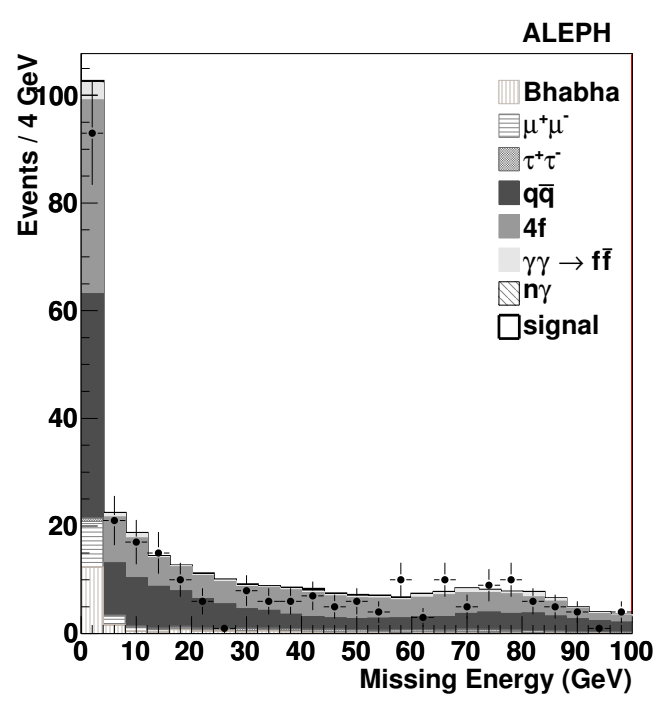

(b)

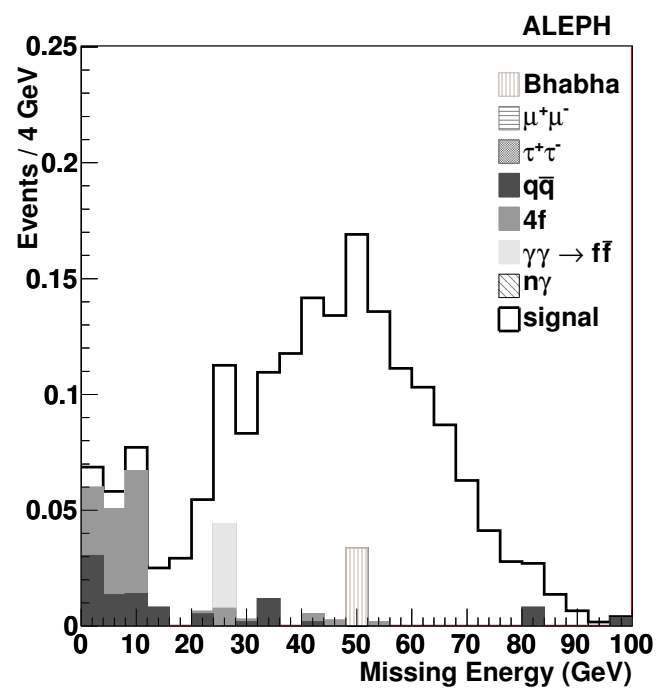

(d)

Figure 2. Distributions for the $\mathrm{Z} \rightarrow \mathrm{e}^{+} \mathrm{e}^{-}$channel after the loose selection for (a) the reconstructed $\mathrm{Z}$ invariant mass and (b) missing energy, where signal corresponds to $m_{\mathrm{h}}=100 \mathrm{GeV} / c^{2}, m_{\mathrm{a}}=$ $4 \mathrm{GeV} / c^{2}$ with $\xi^{2}=1$ (see text). The same distributions are shown in (c) and (d) after the final selection, excluding any requirements on the variable shown.

was chosen to maintain an acceptable signal efficiency for $m_{\mathrm{h}}=86 \mathrm{GeV} / c^{2}$ and $m_{\mathrm{a}}=$ $10 \mathrm{GeV} / c^{2}$. Finally, the second jet was also required to have $n_{2}^{\text {track }}=2$ or 4 . Figure 4 shows the distribution of dijet invariant mass and missing mass for the $\mathrm{Z} \rightarrow \nu \bar{\nu}$ channel. The numbers of events passing loose and final selection in data and simulated background are shown in table 3 . 


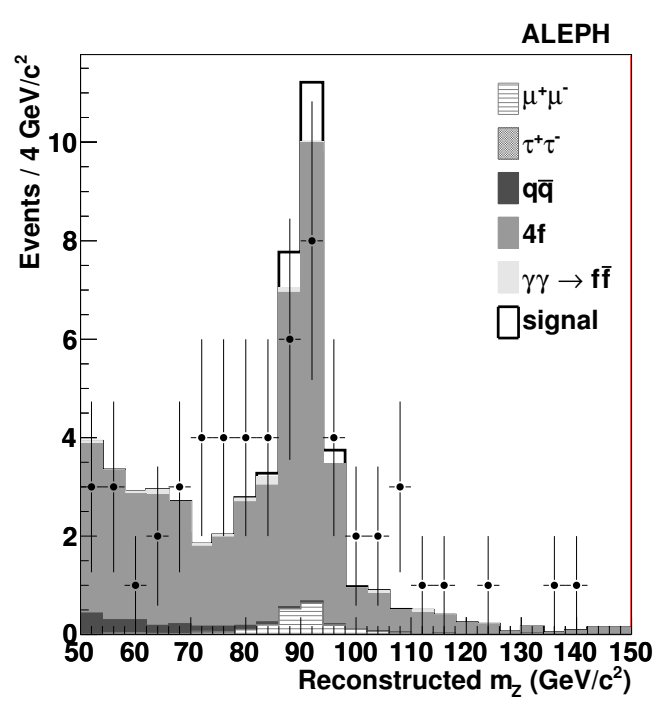

(a)

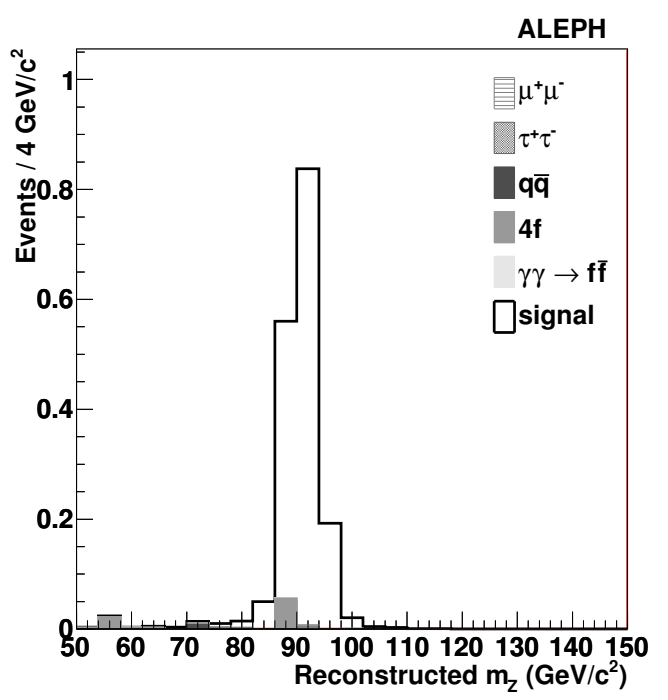

(c)

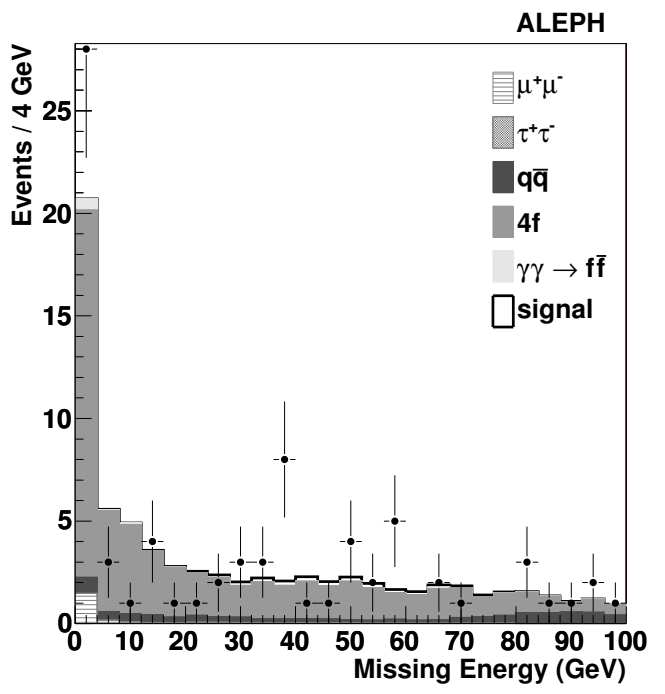

(b)

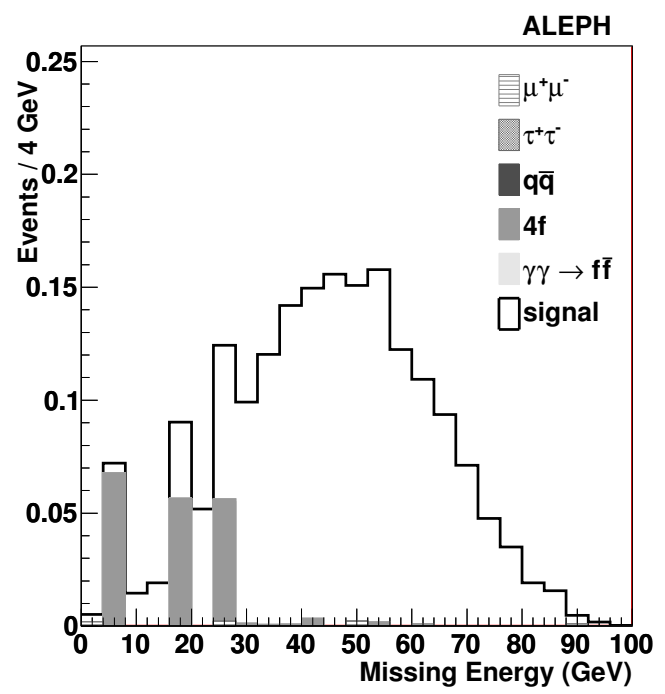

(d)

Figure 3. Distributions for the $\mathrm{Z} \rightarrow \mu^{+} \mu^{-}$channel after the loose selection for (a) the reconstructed $\mathrm{Z}$ invariant mass and (b) missing energy, where signal corresponds to $m_{\mathrm{h}}=100 \mathrm{GeV} / c^{2}, m_{\mathrm{a}}=$ $4 \mathrm{GeV} / c^{2}$ with $\xi^{2}=1$ (see text). The same distributions are shown in (c) and (d) after the final selection, excluding any requirements on the variable shown.

\subsection{Signal efficiency}

The $\mathrm{h} \rightarrow 2 \mathrm{a} \rightarrow 4 \tau$ signal efficiency is shown in figure 5 as a function of the Higgs boson mass with $m_{\mathrm{a}}=4-10 \mathrm{GeV} / c^{2}$ for the three $\mathrm{Z}$ decay channels considered. The decrease in efficiency at higher $m_{\mathrm{a}}$ values, seen in figure 5, is due to two effects. First, the invariant mass of the jet becomes larger and the fraction of three-jet events increases. Second, and more importantly, the events become more aplanar. 


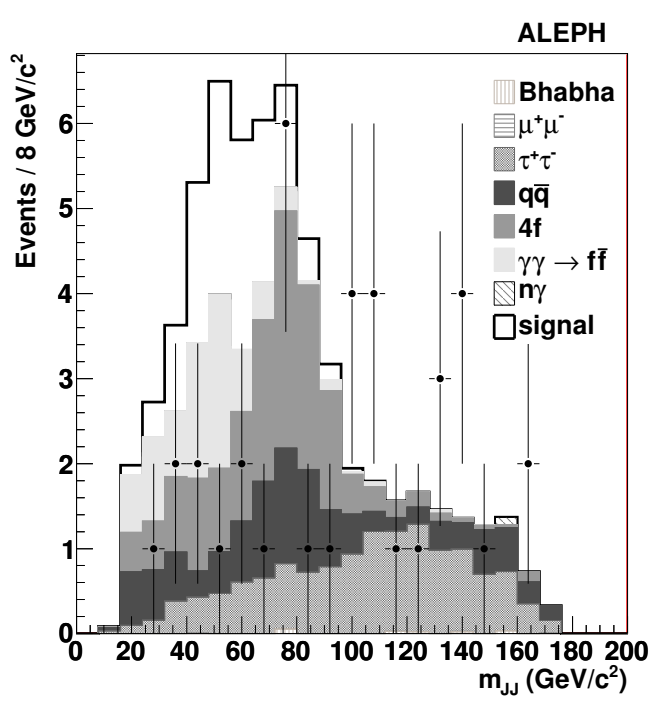

(a)

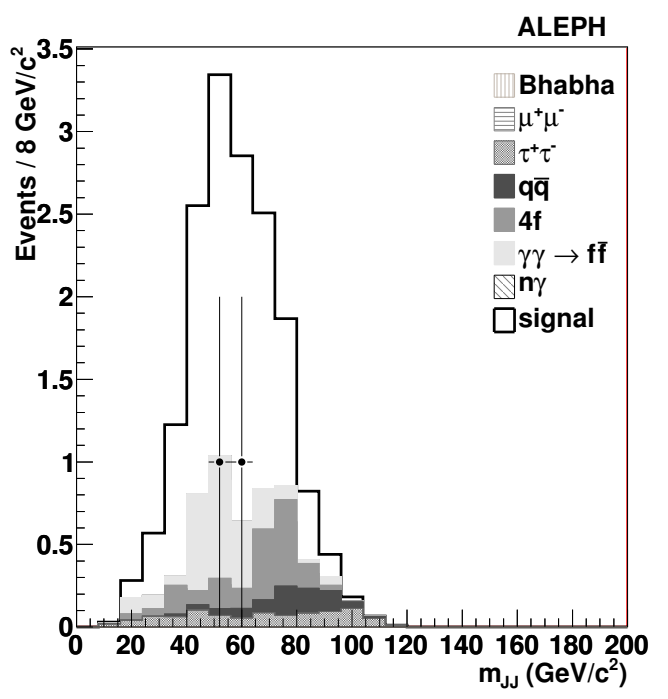

(c)

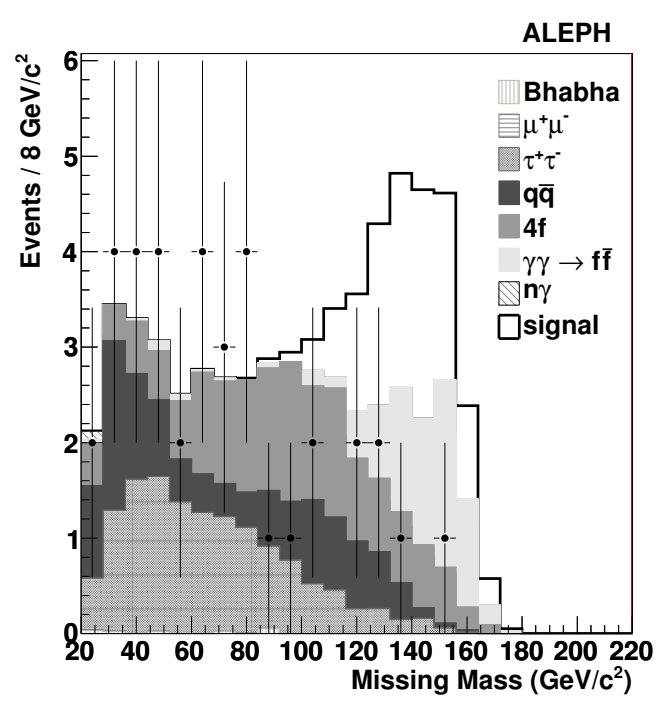

(b)

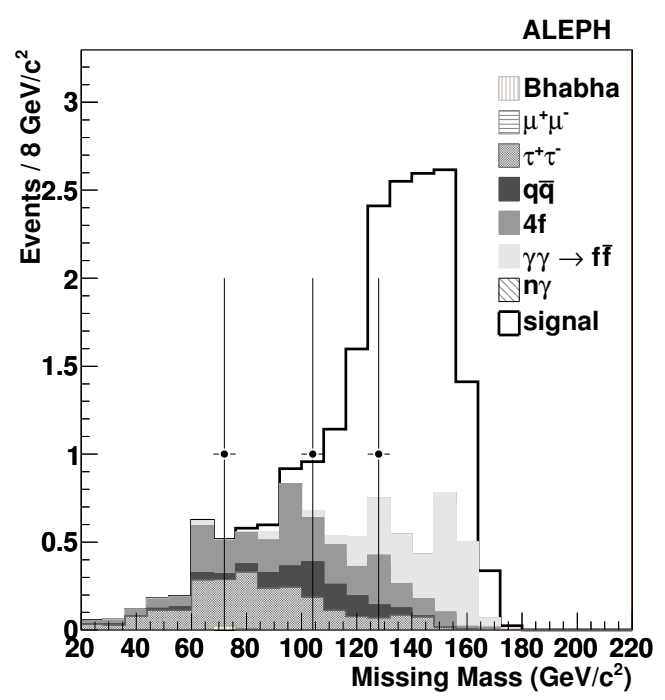

(d)

Figure 4. Distributions for the $\mathrm{Z} \rightarrow \nu \bar{\nu}$ channel after the loose selection and requirement of $1<n_{2}^{\text {track }}<7$ for (a) dijet invariant mass and (b) missing mass, where signal corresponds to $m_{\mathrm{h}}=100 \mathrm{GeV} / c^{2}, m_{\mathrm{a}}=4 \mathrm{GeV} / c^{2}$ with $\xi^{2}=1$ (see text). The same distributions are shown in (c) and (d) after the final selection, excluding any requirements on the variable shown.

\section{Systematic uncertainties}

Uncertainties and inaccuracies in the Monte Carlo simulation lead to systematic effects in the analysis. The impact of uncertainties in jet energy and direction, missing energy, and lepton identification and isolation were estimated in ref. [28] for similar final states. Compared to those, the present analyses do not use neural networks for event selection, do not use $\mathrm{b}$ tagging or tau tagging, and the simulated background samples are substantially larger. The systematic uncertainties related to the simulation of jet energies and directions 


\begin{tabular}{|c|c|c|c|c|c|c|c|c|}
\hline Channel & Selection & data & total & \multicolumn{4}{|c|}{ background category } & signal \\
& $\left(n_{1}^{\text {track }}, n_{2}^{\text {track }}\right)$ & & background & $2 \mathrm{f}$ & $4 \mathrm{f}$ & $\gamma \gamma$ & $\mathrm{n} \gamma$ & \\
\hline \multirow{4}{*}{$\mathrm{Z} \rightarrow \mathrm{e}^{+} \mathrm{e}^{-}$} & Loose & 299 & 332 & 183 & 137 & 12.31 & 0.65 & 2.27 \\
& $(2,2)$ & 0 & 0.034 & 0.034 & 0.000 & 0.000 & 0.000 & 0.689 \\
& $(2,4)+(4,2)$ & 0 & 0.055 & 0.014 & 0.005 & 0.037 & 0.000 & 0.610 \\
& $(4,4)$ & 0 & 0.031 & 0.019 & 0.013 & 0.000 & 0.000 & 0.126 \\
\hline \multirow{5}{*}{$\mathrm{Z} \rightarrow \mu^{+} \mu^{-}$} & Loose & 83 & 74.50 & 12.79 & 60.64 & 1.07 & 0.00 & 2.37 \\
& $(2,2)$ & 0 & 0.058 & 0.005 & 0.053 & 0.000 & 0.000 & 0.800 \\
& $(2,4)+(4,2)$ & 0 & 0.005 & 0.000 & 0.005 & 0.000 & 0.000 & 0.676 \\
& $(4,4)$ & 0 & 0.006 & 0.000 & 0.006 & 0.000 & 0.000 & 0.127 \\
\hline \multirow{3}{*}{$\mathrm{Z} \rightarrow \nu \bar{\nu}$} & Loose & 206 & 200 & 135 & 47.97 & 13.50 & 3.74 & 12.63 \\
& $(2,2)$ & 0 & 1.312 & 0.663 & 0.408 & 0.240 & 0.000 & 5.097 \\
& $(2,4)+(4,2)$ & 0 & 1.948 & 0.528 & 0.575 & 0.845 & 0.000 & 4.741 \\
& $(4,4)$ & 2 & 2.569 & 0.461 & 0.820 & 1.288 & 0.000 & 1.089 \\
\hline
\end{tabular}

Table 3. Number of events passing loose and final selections in each channel, in data, simulated background, and simulated signal $\left(m_{\mathrm{h}}=100 \mathrm{GeV} / c^{2}, m_{\mathrm{a}}=4 \mathrm{GeV} / c^{2}\right)$. The numbers of events passing the final selection are categorised by track multiplicity.

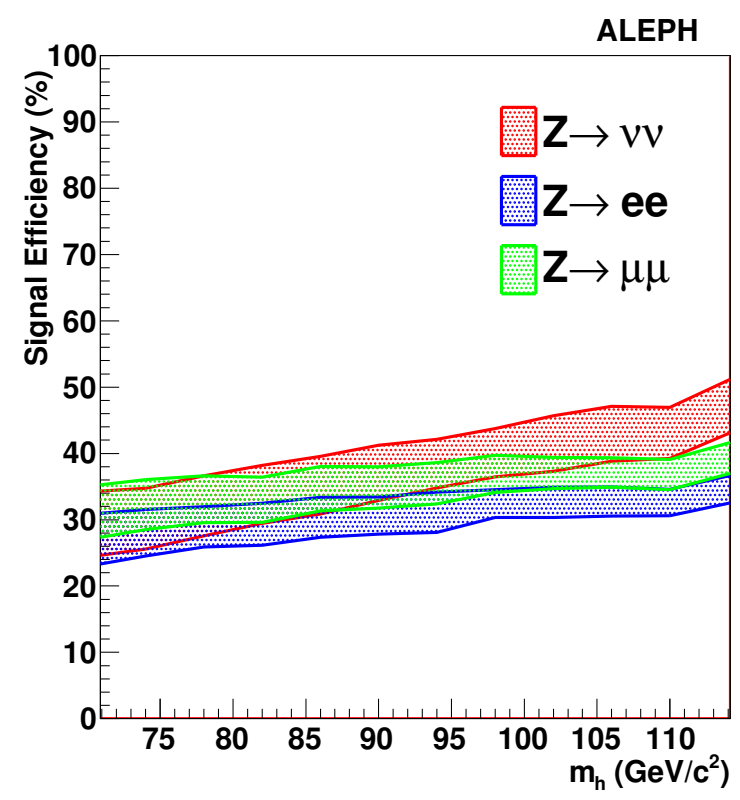

Figure 5. Signal efficiency as a function of the Higgs boson mass for the three channels considered in this work, $\mathrm{Z} \rightarrow \mathrm{e}^{+} \mathrm{e}^{-}, \mu^{+} \mu^{-}$, and $\nu \bar{\nu}$. The upper (lower) portion of the efficiency band corresponds to $m_{\mathrm{a}}=4(10) \mathrm{GeV} / c^{2}$.

were evaluated from the sample of hadronic events collected at the $\mathrm{Z}$ peak in 1998. Based on that sample, it was found that additional smearing of the Monte Carlo simulation was not necessary in the barrel region of the detector. 
For the $\mathrm{Z} \rightarrow \ell^{+} \ell^{-}$channels, the total relative systematic uncertainties from lepton identification and isolation were found to be $0.6 \%, 2.6 \%$ and $7.5 \%$ for the signal, ZZ, and Zee backgrounds, respectively. The systematic uncertainties for WW, We $\nu$, q $\bar{q}$, and other backgrounds were all smaller than $30 \%$. Based on these estimates and the background composition, a $10 \%$ uncertainty is estimated for the background in the $\mathrm{Z} \rightarrow \ell^{+} \ell^{-}$channels.

The cuts used for the $\mathrm{Z} \rightarrow \nu \bar{\nu}$ final state are sensitive to beam related backgrounds. The energy distribution of this background was measured with events recorded at random beam crossings. Additional energy depositions at angles below $12^{\circ}$ were added randomly to all simulated events according to this energy distribution. The relative uncertainty in the total selection efficiency for the analyses presented in ref. [28] was $5 \%$ for the signal and $10 \%$ for ZZ, and it is between $30 \%$ and $100 \%$ for the other background processes. Based on these estimates and the background composition, the uncertainty for the background in the $\mathrm{Z} \rightarrow \nu \bar{\nu}$ channel is estimated to be $30 \%$.

The agreement between the background estimate and the observed number of events in data with the loose selection is within the systematic and statistical uncertainty for all three channels. Given the low numbers of selected events, the final measurements are statistically limited.

\section{Results}

No excess of events above the background was observed. Limits on the cross section times branching ratio with respect to the SM Higgsstrahlung production cross section, $\xi^{2}=$ $\frac{\sigma\left(\mathrm{e}^{+} \mathrm{e}^{-} \rightarrow \mathrm{Zh}\right)}{\sigma_{\mathrm{SM}}\left(\mathrm{e}^{+} \mathrm{e}^{-} \rightarrow \mathrm{Zh}\right)} \times B(\mathrm{~h} \rightarrow \mathrm{aa}) \times B\left(\mathrm{a} \rightarrow \tau^{+} \tau^{-}\right)^{2}$ were determined as follows. A joint probability density was constructed to describe the number of events in each of three jet-multiplicity pairings (indexed by $m$ ) for each of the three final states (indexed by $f$ ). The three jetmultiplicity pairings correspond to the different permutations of one-prong and three-prong tau decays in each of the jets, ignoring those with six tracks in an individual jet, leaving the three permutations $\left(n_{1}^{\text {track }}, n_{2}^{\text {track }}\right) \in\{(2,2),(2,4)$ or $(4,2),(4,4)\} \equiv \mathcal{M}$. The event count $N_{m, f}$ in each of these nine categories was modeled with a Poisson distribution about the sum of the uncertain background $b_{m, f}$ and the expected signal $s_{m, f}$ scaled by $\xi^{2}$. A normal distribution was used to model the relationship between the uncertain background, the Monte Carlo-based background estimate $b_{m, f}^{\mathrm{MC}}$, and its systematic uncertainty $\Delta_{f}$. This procedure leads to the following joint probability density for the event counts:

$$
P\left(N_{m, f} \mid \xi^{2}, b_{m, f}\right)=\prod_{m \in \mathcal{M}} \prod_{f \in\{e e, \mu \mu, \nu \nu\}} \operatorname{Pois}\left(N_{m, f} \mid \xi^{2} s_{m, f}+b_{m, f}\right) \cdot N\left(b_{m, f}^{\mathrm{MC}} \mid b_{m, f}, \Delta_{f}\right) .
$$

Confidence intervals were constructed by using a generalized version of the FeldmanCousins technique [29], which incorporates systematic uncertainties in a frequentist way [30, 31]. Figure 6a shows the $95 \%$ confidence level upper-limit on $\xi^{2}$ as a function of $m_{\mathrm{h}}$ for $m_{\mathrm{a}}=10 \mathrm{GeV} / c^{2}$. Figure $6 \mathrm{~b}$ shows $95 \%$ confidence level contours of $\xi^{2}$ in the $\left(m_{\mathrm{h}}, m_{\mathrm{a}}\right)$ plane. Because the selection has no $m_{\mathrm{h}}$ or $m_{\mathrm{a}}$ dependence, the resulting upper limits are fully correlated. The observed number of events is consistent with a downward fluctuation of the background, which leads to stronger than expected limits on $\xi^{2}$. 


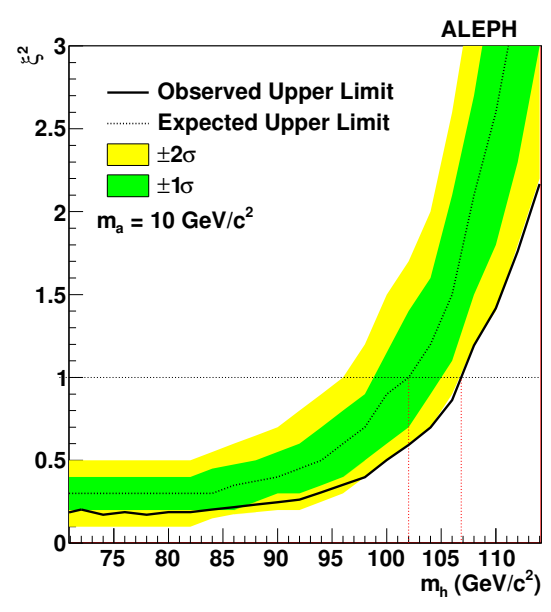

(a)

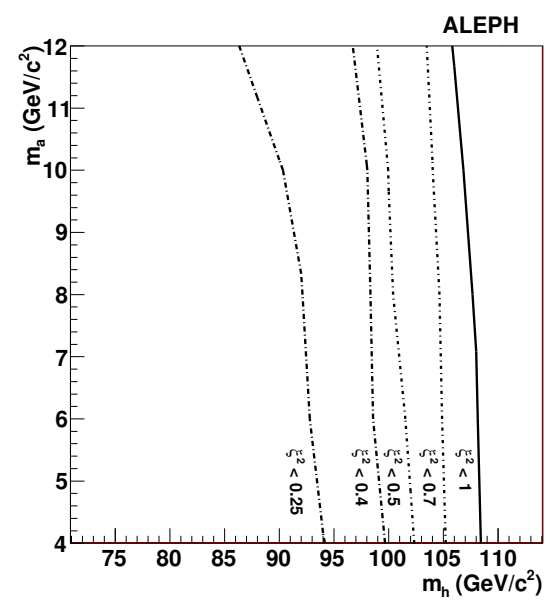

(b)

Figure 6. (a) Observed and expected 95\% confidence level limit on $\xi^{2}$ as a function of the Higgs boson mass for $m_{\mathrm{a}}=10 \mathrm{GeV} / c^{2}$. (b) Contours of observed $95 \%$ confidence level limit on $\xi^{2}$ in the $\left(m_{\mathrm{h}}, m_{\mathrm{a}}\right)$ plane.

\section{Conclusions}

A search for a Higgs boson produced via Higgsstrahlung at LEP2 energies has been performed, where $\mathrm{h} \rightarrow 2 \mathrm{a} \rightarrow 4 \tau$ and $\mathrm{Z} \rightarrow \mathrm{e}^{+} \mathrm{e}^{-}, \mu^{+} \mu^{-}, \nu \bar{\nu}$. No evidence for an excess of events above background was observed, and a limit on the combined production cross section times branching ratio, $\xi^{2}=\frac{\sigma\left(\mathrm{e}^{+} \mathrm{e}^{-} \rightarrow \mathrm{Zh}\right)}{\sigma_{\mathrm{SM}}\left(\mathrm{e}^{+} \mathrm{e}^{-} \rightarrow \mathrm{Zh}\right)} \times B(\mathrm{~h} \rightarrow \mathrm{aa}) \times B\left(\mathrm{a} \rightarrow \tau^{+} \tau^{-}\right)^{2}$ is presented. For $m_{\mathrm{h}}<107 \mathrm{GeV} / c^{2}$ and $4<m_{\mathrm{a}}<10 \mathrm{GeV} / c^{2}, \xi^{2}>1$ is excluded at the $95 \%$ confidence level. This analysis covers a region of parameter space previously left unexplored, and further constrains models with non-standard Higgs decays, such as the NMSSM.

\section{Acknowledgments}

We wish to thank Neal Weiner and Riccardo Barbieri for providing encouragement, motivation, and advice throughout this work. It is a pleasure to congratulate our colleagues from the CERN accelerator divisions for the successful operation of LEP throughout the LEP2 years. We are indebted to the engineers and technicians in all our institutions for their contributions to the excellent performance of ALEPH. Those of us from non-member countries thank CERN for its hospitality. 
Author list

\section{S. Schael}

Physikalisches Institut der RWTH-Aachen, D-52056 Aachen, Germany

R. Barate, R. Brunelière, I. De Bonis, D. Decamp, C. Goy, S. Jézéquel, J.-P. Lees, F. Martin, E. Merle, M.-N. Minard, B. Pietrzyk and B. Trocmé Laboratoire de Physique des Particules (LAPP), IN ${ }^{1} P^{2}$-CNRS, F-74019 Annecy-le-Vieux Cedex, France

S. Bravo, M.P. Casado, M. Chmeissani, J.M. Crespo, E. Fernandez, M. Fernandez-Bosman, Ll. Garrido, ${ }^{3}$ M. Martinez, A. Pacheco and H. Ruiz Institut de Física d'Altes Energies, Universitat Autònoma de Barcelona, E-08193 Bellaterra, Barcelona, Spain ${ }^{4}$

A. Colaleo, D. Creanza, N. De Filippis, M. de Palma, G. Iaselli, G. Maggi, M. Maggi, S. Nuzzo, A. Ranieri, G. Raso, ${ }^{5}$ F. Ruggieri, G. Selvaggi, L. Silvestris, P. Tempesta, A. Tricomi ${ }^{6}$ and G. Zito

Dipartimento di Fisica, INFN Sezione di Bari,

I-70126 Bari, Italy

X. Huang, J. Lin, Q. Ouyang, T. Wang, Y. Xie, R. Xu, S. Xue, J. Zhang, L. Zhang and W. Zhao

Institute of High Energy Physics, Academia Sinica, Beijing, The People's Republic of China ${ }^{7}$

D. Abbaneo, T. Barklow ${ }^{8}$ O. Buchmüller, ${ }^{9}$ M. Cattaneo, B. Clerbaux, ${ }^{10}$ H. Drevermann, R.W. Forty, M. Frank, F. Gianotti, J.B. Hansen, J. Harvey, D.E. Hutchcroft, ${ }^{11}$ P. Janot, B. Jost, M. Kado, ${ }^{12}$ P. Mato, A. Moutoussi, F. Ranjard, L. Rolandi, D. Schlatter, F. Teubert, A. Valassi and I. Videau European Laboratory for Particle Physics (CERN),

CH-1211 Geneva 23, Switzerland

\footnotetext{
${ }^{1}$ Now at Fermilab, PO Box 500, MS 352, Batavia, IL 60510, U.S.A.

${ }^{2}$ Also at Dipartimento di Fisica di Catania and INFN Sezione di Catania, 95129 Catania, Italy.

${ }^{3}$ Permanent address: Universitat de Barcelona, 08208 Barcelona, Spain.

${ }^{4}$ Supported by CICYT, Spain.

${ }^{5}$ Now at Dipartimento di Fisica e Tecnologie Relative, Università di Palermo, Palermo, Italy.

${ }^{6}$ Also at Dipartimento di Fisica di Catania and INFN Sezione di Catania, 95129 Catania, Italy.

${ }^{7}$ Supported by the National Science Foundation of China.

${ }^{8}$ Now at SLAC, Stanford, CA 94309, U.S.A.

${ }^{9}$ Now at SLAC, Stanford, CA 94309, U.S.A.

${ }^{10}$ Now at IIHE, CP 230, Université Libre de Bruxelles, 1050 Bruxelles, Belgique.

${ }^{11}$ Now at Liverpool University, Liverpool L69 7ZE, United Kingdom.

${ }^{12}$ Now at Fermilab, PO Box 500, MS 352, Batavia, IL 60510, U.S.A.
} 
F. Badaud, S. Dessagne, A. Falvard, ${ }^{13}$ D. Fayolle, P. Gay, J. Jousset, B. Michel, S. Monteil, D. Pallin, J.M. Pascolo and P. Perret

Laboratoire de Physique Corpusculaire, Université Blaise Pascal, IN ${ }^{14} P^{15}$-CNRS, Clermont-Ferrand, F-63177 Aubière, France

J.D. Hansen, J.R. Hansen, P.H. Hansen, A.C. Kraan and B.S. Nilsson Niels Bohr Institute, 2100 Copenhagen, Denmark ${ }^{16}$

A. Kyriakis, C. Markou, E. Simopoulou, A. Vayaki and K. Zachariadou Nuclear Research Center Demokritos (NRCD), GR-15310 Attiki, Greece

A. Blondel, ${ }^{17}$ J.-C. Brient, F. Machefert, A. Rougé and H. Videau Laoratoire Leprince-Ringuet, Ecole Polytechnique, IN ${ }^{18} P^{19}$-CNRS, F-91128 Palaiseau Cedex, France

V. Ciulli, E. Focardi and G. Parrini

Dipartimento di Fisica, Università di Firenze, INFN Sezione di Firenze, I-50125 Firenze, Italy

A. Antonelli, M. Antonelli, G. Bencivenni, F. Bossi, G. Capon, F. Cerutti, V. Chiarella, P. Laurelli, G. Mannocchi, ${ }^{20}$ G.P. Murtas and L. Passalacqua Laboratori Nazionali dell'INFN (LNF-INFN),

I-00044 Frascati, Italy

J. Kennedy, J.G. Lynch, P. Negus, V. O'Shea and A.S. Thompson

Department of Physics and Astronomy, University of Glasgow, Glasgow G12, 8QQ, United Kingdom ${ }^{21}$

\section{S. Wasserbaech}

Utah Valley State College,

Orem, UT 84058, U.S.A.

\footnotetext{
${ }^{13}$ Now at Groupe d' Astroparticules de Montpellier, Université de Montpellier II, Montpellier, France.

${ }^{14}$ Now at Fermilab, PO Box 500, MS 352, Batavia, IL 60510, U.S.A.

${ }^{15}$ Also at Dipartimento di Fisica di Catania and INFN Sezione di Catania, 95129 Catania, Italy.

${ }^{16}$ Supported by the Danish Natural Science Research Council.

${ }^{17}$ Supported by the Danish Natural Science Research Council.

${ }^{18}$ Now at Fermilab, PO Box 500, MS 352, Batavia, IL 60510, U.S.A.

${ }^{19}$ Also at Dipartimento di Fisica di Catania and INFN Sezione di Catania, 95129 Catania, Italy.

${ }^{20}$ Also IFSI sezione di Torino, INAF, Italy.

${ }^{21}$ Supported by the U.K. Particle Physics and Astronomy Research Council.
} 
R. Cavanaugh, ${ }^{22}$ S. Dhamotharan, ${ }^{23}$ C. Geweniger, P. Hanke, V. Hepp, E.E. Kluge, A. Putzer, H. Stenzel, K. Tittel and M. Wunsch ${ }^{24}$

Kirchhoff-Institut für Physik, Universität Heidelberg,

D-69120 Heidelberg, Germany ${ }^{25}$

R. Beuselinck, W. Cameron, G. Davies, P.J. Dornan, M. Girone, ${ }^{26}$ N. Marinelli, J. Nowell, S.A. Rutherford, J.K. Sedgbeer, J.C. Thompson ${ }^{27}$ and R. White Department of Physics, Imperial College,

London SW7 2BZ, United Kingdom ${ }^{28}$

V.M. Ghete, P. Girtler, E. Kneringer, D. Kuhn and G. Rudolph Institut für Experimentalphysik, Universität Innsbruck,

A-6020 Innsbruck, Austria ${ }^{29}$

E. Bouhova-Thacker, C.K. Bowdery, D.P. Clarke, G. Ellis, A.J. Finch, F. Foster, G. Hughes, R.W.L. Jones, M.R. Pearson, N.A. Robertson, T. Sloan and M. Smizanska

Department of Physics, University of Lancaster,

Lancaster LA1 4YB, United Kingdom ${ }^{30}$

O. van der Aa, C. Delaere, ${ }^{31}$ G.Leibenguth ${ }^{32}$ and V. Lemaitre ${ }^{33}$

Institut de Physique Nucléaire, Département de Physique,

Université Catholique de Louvain,

1348 Louvain-la-Neuve, Belgium

U. Blumenschein, F. Hölldorfer, K. Jakobs, F. Kayser, A.-S. Müller, B. Renk, H.-G. Sander, S. Schmeling, H. Wachsmuth, C. Zeitnitz and T. Ziegler Institut für Physik, Universität Mainz,

D-55099 Mainz, Germany ${ }^{34}$

A. Bonissent, P. Coyle, C. Curtil, A. Ealet, D. Fouchez, P. Payre and A. Tilquin Centre de Physique des Particules de Marseille, Univ Méditerranée, IN ${ }^{35} P^{36}$-CNRS, F-13288 Marseille, France

\footnotetext{
${ }^{22}$ Now at University of Florida, Department of Physics, Gainesville, Florida 32611-8440, U.S.A.

${ }^{23}$ Now at BNP Paribas, 60325 Frankfurt am Mainz, Germany.

${ }^{24}$ Now at SAP AG, 69185 Walldorf, Germany.

${ }^{25}$ Supported by Bundesministerium für Bildung und Forschung, Germany.

${ }^{26}$ Also at CERN, 1211 Geneva 23, Switzerland.

${ }^{27}$ Supported by the Leverhulme Trust.

${ }^{28}$ Supported by the U.K. Particle Physics and Astronomy Research Council.

${ }^{29}$ Supported by the Austrian Ministry for Science and Transport.

${ }^{30}$ Supported by the U.K. Particle Physics and Astronomy Research Council.

${ }^{31}$ Research Fellow of the Belgium FNRS.

${ }^{32}$ Supported by the Interuniversity Attraction Pole P5/27.

${ }^{33}$ Research Associate of the Belgium FNRS.

${ }^{34}$ Supported by Bundesministerium für Bildung und Forschung, Germany.

${ }^{35}$ Now at Fermilab, PO Box 500, MS 352, Batavia, IL 60510, U.S.A.

${ }^{36}$ Also at Dipartimento di Fisica di Catania and INFN Sezione di Catania, 95129 Catania, Italy.
} 


\section{F. Ragusa}

Dipartimento di Fisica, Università di Milano e INFN Sezione di Milano, I-20133 Milano, Italy
A. David, H. Dietl, ${ }^{37}$ G. Ganis, ${ }^{38}$ K. Hüttmann, G. Lütjens, W. Männer, ${ }^{39}$ H.-G. Moser, R. Settles, M. Villegas and G. Wolf
Max-Planck-Institut für Physik, Werner-Heisenberg-Institut, D-80805 München, Germany ${ }^{40}$

\section{J. Beacham, K. Cranmer ${ }^{41}$ and I. Yavin ${ }^{42}$}

Center for Cosmology and Particle Physics, New York University,

New York, NY 10003, U.S.A.

J. Boucrot, O. Callot, M. Davier, L. Duflot, J.-F. Grivaz, Ph. Heusse, A. Jacholkowska, ${ }^{43}$ L. Serin and J.-J. Veillet

Laboratoire de l'Accélérateur Linéaire, Université de Paris-Sud, $I N^{44} P^{45}$ _CNRS, F-91898 Orsay Cedex, France

P. Azzurri, G. Bagliesi, T. Boccali, L. Foà, A. Giammanco, A. Giassi, F. Ligabue, A. Messineo, F. Palla, G. Sanguinetti, A. Sciabà, G. Sguazzoni, P. Spagnolo, R. Tenchini, A. Venturi and P.G. Verdini

Dipartimento di Fisica dell'Università, INFN Sezione di Pisa e Scuola Normale Superiore, I-56010 Pisa, Italy

O. Awunor, G.A. Blair, G. Cowan, A. Garcia-Bellido, M.G. Green, T. Medcalf, ${ }^{46}$ A. Misiejuk, J.A. Strong ${ }^{47}$ and P. Teixeira-Dias

Department of Physics, Royal Holloway \& Bedford New College, University of London, Egham, Surrey TW20 OEX, United Kingdom ${ }^{48}$

R.W. Clifft, T.R. Edgecock, P.R. Norton, I.R. Tomalin and J.J. Ward Particle Physics Dept., Rutherford Appleton Laboratory, Chilton, Didcot, Oxon OX11 OQX, United Kingdom ${ }^{49}$

\footnotetext{
${ }^{37}$ Now at Henryk Niewodnicznski Institute of Nuclear Physics, Cracow, Poland.

${ }^{38}$ Now at CERN, 1211 Geneva 23, Switzerland.

${ }^{39}$ Now at Henryk Niewodnicznski Institute of Nuclear Physics, Cracow, Poland.

${ }^{40}$ Supported by Bundesministerium für Bildung und Forschung, Germany.

${ }^{41}$ Supported by US National Science Foundation grant PHY-0854724.

42 Supported by the James Arthur fellowship.

${ }^{43}$ Also at Groupe d'Astroparticules de Montpellier, Université de Montpellier II, Montpellier, France.

${ }^{44}$ Now at Fermilab, PO Box 500, MS 352, Batavia, IL 60510, U.S.A.

${ }^{45}$ Also at Dipartimento di Fisica di Catania and INFN Sezione di Catania, 95129 Catania, Italy.

${ }^{46}$ Deceased.

${ }^{47}$ Deceased.

${ }^{48}$ Supported by the UK Particle Physics and Astronomy Research Council.

${ }^{49}$ Supported by the UK Particle Physics and Astronomy Research Council.
} 
B. Bloch-Devaux, D. Boumediene, P. Colas, B. Fabbro, E. Lançon, M.C. Lemaire, E. Locci, P. Perez, J. Rander, B. Tuchming and B. Vallage CEA, DAPNIA/Service de Physique des Particules, CE-Saclay, F-91191 Gif-sur-Yvette Cedex, France ${ }^{50}$

A.M. Litke and G. Taylor

Institute for Particle Physics, University of California at Santa Cruz,

Santa Cruz, CA 95064, U.S.A. ${ }^{51}$

C.N. Booth, S. Cartwright, F. Combley, ${ }^{52}$ P.N. Hodgson, M. Lehto and L.F. Thompson

Department of Physics, University of Sheffield,

Sheffield S3 7RH, United Kingdom ${ }^{53}$

A. Böhrer, S. Brandt, C. Grupen, J. Hess, A. Ngac and G. Prange

Fachbereich Physik, Universität Siegen,

D-57068 Siegen, Germany ${ }^{54}$

C. Borean and G. Giannini

Dipartimento di Fisica, Università di Trieste e INFN Sezione di Trieste,

I-34127 Trieste, Italy

H. He, J. Putz and J. Rothberg

Experimental Elementary Particle Physics, University of Washington,

Seattle, WA 98195 U.S.A.

S.R. Armstrong, K. Berkelman, D.P.S. Ferguson, Y. Gao, ${ }^{55}$ S. González, O.J. Hayes, H. Hu, S. Jin, J. Kile, P.A. McNamara III, J. Nielsen, Y.B. Pan, J.H. von Wimmersperg-Toeller, W. Wiedenmann, J. Wu, Sau Lan Wu, X. Wu and G. Zobernig

Department of Physics, University of Wisconsin,

Madison, WI 53706, U.S.A. ${ }^{56}$

\section{G. Dissertori}

Institute for Particle Physics,

ETH Hönggerberg, 8093 Zürich, Switzerland

\footnotetext{
${ }^{50}$ Supported by the Direction des Sciences de la Matière, C.E.A.

${ }^{51}$ Supported by the US Department of Energy, grant DE-FG03-92ER40689.

${ }^{52}$ Deceased.

${ }^{53}$ Supported by the UK Particle Physics and Astronomy Research Council.

${ }^{54}$ Supported by Bundesministerium für Bildung und Forschung, Germany.

${ }^{55}$ Also at Department of Physics, Tsinghua University, Beijing, The People's Republic of China.

${ }^{56}$ Supported by the US Department of Energy, grant DE-FG0295-ER40896.
} 
Open Access. This article is distributed under the terms of the Creative Commons Attribution Noncommercial License which permits any noncommercial use, distribution, and reproduction in any medium, provided the original author(s) and source are credited.

\section{References}

[1] LEP Working Group for Higgs boson searches collaboration, R. Barate et al., Search for the standard model Higgs boson at LEP, Phys. Lett. B 565 (2003) 61 [hep-ex/0306033] [SPIRES].

[2] ALEPH collaboration, S. Schael et al., Search for neutral MSSM Higgs bosons at LEP, Eur. Phys. J. C 47 (2006) 547 [hep-ex/0602042] [SPIRES].

[3] R. Dermisek and J.F. Gunion, Consistency of LEP event excesses with an $h \rightarrow$ a a decay scenario and low-fine-tuning NMSSM models, Phys. Rev. D 73 (2006) 111701 [hep-ph/0510322] [SPIRES].

[4] R. Barbieri and A. Strumia, What is the limit on the Higgs mass?, Phys. Lett. B 462 (1999) 144 [hep-ph/9905281] [SPIRES].

[5] R. Dermisek and J.F. Gunion, Escaping the large fine tuning and little hierarchy problems in the next to minimal supersymmetric model and $h \rightarrow a$ a decays, Phys. Rev. Lett. 95 (2005) 041801 [hep-ph/0502105] [SPIRES].

[6] R. Dermisek and J.F. Gunion, The NMSSM solution to the fine-tuning problem, precision electroweak constraints and the largest LEP Higgs event excess, Phys. Rev. D 76 (2007) 095006 [arXiv:0705.4387] [SPIRES].

[7] S. Chang, P.J. Fox and N. Weiner, Naturalness and Higgs decays in the MSSM with a singlet, JHEP 08 (2006) 068 [hep-ph/0511250] [SPIRES].

[8] S. Chang, R. Dermisek, J.F. Gunion and N. Weiner, Nonstandard Higgs boson decays, Ann. Rev. Nucl. Part. Sci. 58 (2008) 75 [arXiv:0801.4554] [SPIRES].

[9] OPAL collaboration, G. Abbiendi et al., Search for neutral Higgs boson in CP-conserving and CP-violating MSSM scenarios, Eur. Phys. J. C 37 (2004) 49 [hep-ex/0406057] [SPIRES].

[10] DELPHI collaboration, J. Abdallah et al., Higgs boson searches in CP-conserving and CP-violating MSSM scenarios with the DELPHI detector, Eur. Phys. J. C 54 (2008) 1 [Erratum ibid. C 56 (2008) 165] [arXiv:0801.3586] [SPIRES].

[11] OPAL collaboration, G. Abbiendi et al., Search for a low mass CP-odd Higgs boson in $e^{+} e^{-}$ collisions with the OPAL detector at LEP2, Eur. Phys. J. C 27 (2003) 483 [hep-ex/0209068] [SPIRES].

[12] D0 collaboration, V.M. Abazov et al., Search for NMSSM Higgs bosons in the

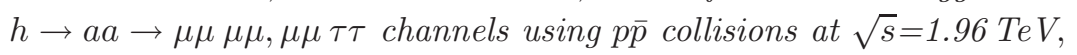
Phys. Rev. Lett. 103 (2009) 061801 [arXiv:0905. 3381] [SPIRES].

[13] ALEPH collaboration, D. Decamp et al., ALEPH: a detector for electron-positron annihilations at LEP, Nucl. Instrum. Meth. A 294 (1990) 121 [Erratum ibid. A 303 (1991) 393] [SPIRES].

[14] ALEPH collaboration, D. Buskulic et al., Performance of the ALEPH detector at LEP, Nucl. Instrum. Meth. A 360 (1995) 481 [SPIRES]. 
[15] R. Brun et al., Geant3, CERN Internal Report No. CERN DD/EE/84-1 (1987) [SPIRES].

[16] G. Ganis and P. Janot, The HZHA generator, Physics at LEP, G. Altarelli, T. Sjöstrand and F. Zwirner eds., CERN 96-01 vol. 2 (1996), pg. 309.

[17] T. Sjöstrand et al., High-energy physics event generation with PYTHIA 6.1, Comput. Phys. Commun. 135 (2001) 238 [hep-ph/0010017] [SPIRES].

[18] E. Barberio and Z. Was, PHOTOS: a universal Monte Carlo for QED radiative corrections. Version 2.0, Comput. Phys. Commun. 79 (1994) 291 [SPIRES].

[19] S. Jadach, Z. Was, R. Decker and J.H. Kuhn, The $\tau$ decay library TAUOLA: Version 2.4, Comput. Phys. Commun. 76 (1993) 361 [SPIRES].

[20] ALEPH collaboration, A. Heister et al., Measurement of $W$ pair production in $e^{+} e^{-}$ collisions at centre-of-mass energies from 183-GeV to 209-GeV, Eur. Phys. J. C 38 (2004) 147 [SPIRES].

[21] S. Jadach, B.F.L. Ward and Z. Was, The precision Monte Carlo event generator KK for twofermion final states in $e^{+} e^{-}$collisions, Comput. Phys. Commun. 130 (2000) 260 [hep-ph/9912214] [SPIRES].

[22] S. Jadach, W. Placzek and B.F.L. Ward, BHWIDE 1.00: $O(\alpha)$ YFS exponentiated Monte Carlo for Bhabha scattering at wide angles for LEP1/SLC and LEPQ, Phys. Lett. B 390 (1997) 298 [hep-ph/9608412] [SPIRES].

[23] S. Jadach, W. Placzek, M. Skrzypek, B.F.L. Ward and Z. Was, The Monte Carlo program KoralW version 1.51 and the concurrent Monte Carlo KoralW 83 YFSWW3 with all background graphs and first order corrections to $W$ pair production, Comput. Phys. Commun. 140 (2001) 475 [hep-ph/0104049] [SPIRES].

[24] J.A.M. Vermaseren, $2 \gamma$ physics versus $1 \gamma$ physics and whatever lies in between, proceedings of the IV International Workshop on Gamma Gamma Interactions, Amiens, France, G. Cochard and P. Kessler eds. (1980).

[25] ALEPH collaboration, D. Buskulic et al., An experimental study of $\gamma \gamma \rightarrow$ hadrons at LEP, Phys. Lett. B 313 (1993) 509 [SPIRES].

[26] JADE collaboration, W. Bartel et al., Determination of semimuonic branching ratios and fragmentation functions of heavy quarks in $e^{+} e^{-}$annihilation at $\sqrt{s}=34.6-\mathrm{GeV}$, Z. Phys. C 33 (1987) 339 [SPIRES].

[27] JADE collaboration, S. Bethke et al., Experimental investigation of the energy dependence of the strong coupling strength, Phys. Lett. B 213 (1988) 235 [SPIRES].

[28] ALEPH collaboration, R. Barate et al., Search for the neutral Higgs bosons of the standard model and the MSSM in $e^{+} e^{-}$collisions at $\sqrt{s}=189$ GeV, Eur. Phys. J. C 17 (2000) 223 [SPIRES].

[29] G.J. Feldman and R.D. Cousins, A unified approach to the classical statistical analysis of small signals, Phys. Rev. D 57 (1998) 3873 [physics/9711021] [SPIRES].

[30] T. L. Chuang, C-S. Lai, Resampling methods for confidence intervals in group sequential trials, Biometrika 85 (1998) 317.

[31] K. Cranmer, Statistical challenges for searches for new physics at the LHC, proceedings of PhyStat05: Statistical Problems in Particle Physics, Astrophysics and Cosmology, Oxford, England, U.K. (2005) [SPIRES]. 